\title{
Magnetic Properties of Finite Systems: Microcanonical Finite-Size Scaling
}

\author{
Michael Promberger, Michael Kastner, and Alfred Hüller \\ Institut für Theoretische Physik, Staudtstraße 7B3, D-91058 Erlangen, Germany
}

\begin{abstract}
In the microcanonical ensemble, suitably defined observables show nonanalyticities and power law behaviour even for finite systems. For these observables, a microcanonical finite-size scaling theory is established which facilitates an approach to the critical exponents of the infinite system.
\end{abstract}

\section{Introduction: microcanonical description of finite systems}

In a microcanonical description of finite systems, the magnetic equation of state and the zero-field isothermal magnetic susceptibility are defined in terms of derivatives of the microcanonical entropy $s\left(\varepsilon, m, L^{-1}\right)$ (cf. [2], 3]):

$$
\begin{aligned}
& m_{h=0}\left(\varepsilon, L^{-1}\right) \Longleftarrow \max _{m \geq 0}\left\{s\left(\varepsilon, m, L^{-1}\right)\right\}=s\left(\varepsilon, m_{h=0}\left(\varepsilon, L^{-1}\right), L^{-1}\right) \\
& \chi_{T ; h=0}\left(\varepsilon, L^{-1}\right):=\left.\frac{\partial m}{\partial h}\right|_{T ; h=0}\left(\varepsilon, L^{-1}\right)= \\
& =\left\{\left[\frac{\partial s}{\partial \varepsilon}\left[\left(\frac{\partial^{2} s}{\partial \varepsilon \partial m}\right)^{2} / \frac{\partial^{2} s}{\partial \varepsilon^{2}}-\frac{\partial^{2} s}{\partial m^{2}}\right]^{-1}\right]\left(\varepsilon, m, L^{-1}\right)\right\}_{m=m_{h=0}\left(\varepsilon, L^{-1}\right)}
\end{aligned}
$$

Here, $\varepsilon$ denotes the specific enthalpy (cf. [1]), $h$ the magnetic field, $m$ the specific magnetization, $L$ the linear system size and $d$ the spatial dimension. For notational convenience, the zero field limit is denoted by $h=0$. By definition, the microcanonical entropy is the logarithm of the microcanonical partition function (density of states) $\Omega$ :

$$
s\left(\varepsilon, m, L^{-1}\right)=L^{-d} \ln \Omega\left(\varepsilon, m, L^{-1}\right) .
$$

In Fig. 1, the zero-field magnetization (Fig. 1a) and the zero-field magnetic susceptibility (Fig. 1b) are plotted for the example of the $3 d$-Ising system for various system sizes. Both observables show a behaviour reminiscent of the critical behaviour of the infinite system and therefore suggest the introduction of finite system exponents $\beta_{\varepsilon, L}$ and $\gamma_{\varepsilon, L}$ (describing the power law behaviour with respect to the base $|\tilde{\varepsilon}|$ defined below; cf. Ref. 13 of [2]). 
The transition energies $\varepsilon_{T}(L)$, at which the non-analyticities occur, are obviously dependent on system size.

In Figs. 1c/d, magnetizations and susceptibilities are plotted as functions of the reduced enthalpy $\tilde{\varepsilon}(L):=\left(\varepsilon-\varepsilon_{T}(L)\right) /\left|\varepsilon_{T}(L)\right|$.

a) $\quad m_{h=0}\left(\varepsilon, L^{-1}\right)$ vs. $\varepsilon$

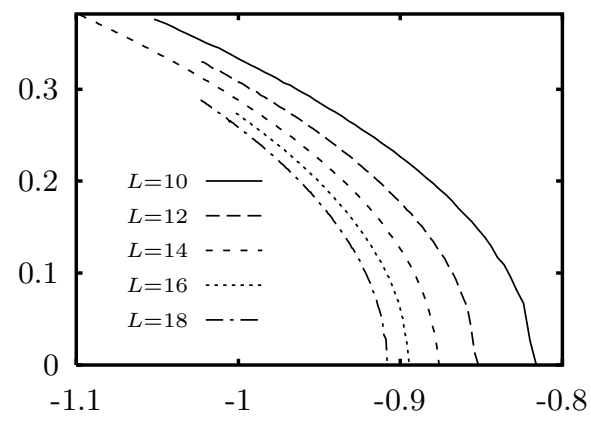

c) $\quad A\left(L^{-1}\right) m_{h=0}\left(\tilde{\varepsilon}, L^{-1}\right)$ vs. $\tilde{\varepsilon}$

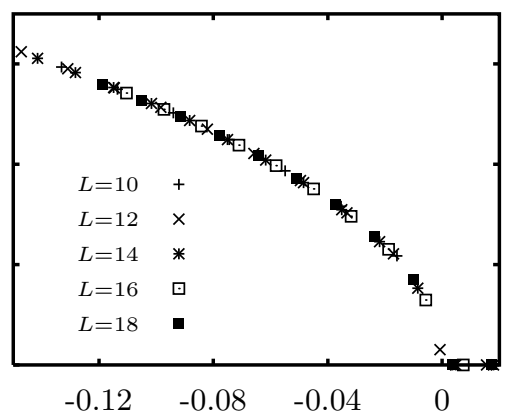

b) $\left[\frac{\partial^{2} s}{\partial m^{2}}\right]^{-1}\left(\varepsilon, m_{h=0}, L^{-1}\right)$ vs. $\varepsilon$

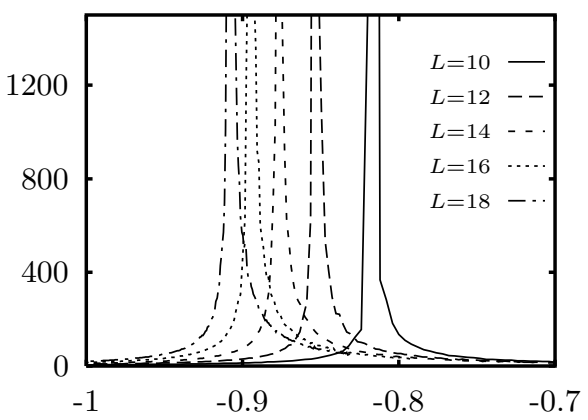

d) $B\left(L^{-1}\right)\left[\frac{\partial^{2} s}{\partial m^{2}}\right]\left(\tilde{\varepsilon}, m_{h=0}, L^{-1}\right)$ vs. $\tilde{\varepsilon}$

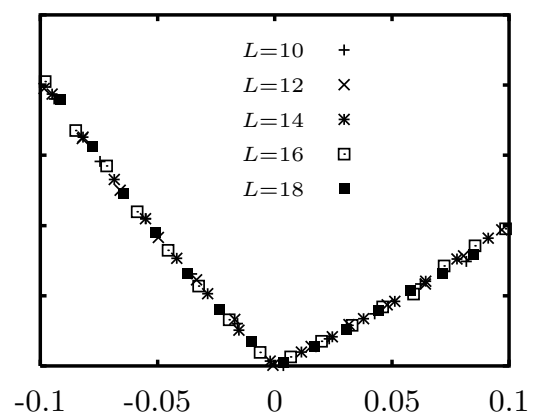

Fig. 1. Microcanonical magnetizations and "susceptibilities" of finite $3 d$-Ising systems (Figs. a/b). For numerical convenience, $\left[\left(\partial^{2} s\right) /\left(\partial m^{2}\right)\right]^{-1}$ is shown instead of $\chi_{T ; h=0}$. Note that both quantities show the same microcanonical finite-size scaling behaviour and - in the thermodynamic limit - the same critical behaviour. Multiplication of $m_{h=0}$ and $\left(\partial^{2} s\right) /\left(\partial m^{2}\right)$ by suitable scaling factors $A\left(L^{-1}\right)$ and $B\left(L^{-1}\right)$ and plotting the thus obtained results against $\tilde{\varepsilon}$ yields data collapse (Figs. c/d). Whereas Figs. a/b suggest the introduction of finite system exponents, Figs. c/d give rise to the assumption that the thus defined exponents show no system size dependence.

Data collapse is achieved by suitable scaling of the amplitudes of $m_{h=0}$ and $\chi_{T ; h=0}$. Therefore, it seems to be evident that the finite system critical exponents are independent of the system size (i.e. take on the same value 
$\forall L^{-1} \neq 0$ ), but differ from the values expected in the thermodynamic limit (TDL), $\beta_{\varepsilon, \infty} \approx 0.37, \gamma_{\varepsilon, \infty} \approx 1.38$, whereas from numerical data we obtain $\beta_{\varepsilon, L} \approx 0.5, \gamma_{\varepsilon, L} \approx 1$ for all $L$ considered. In this paper, a microcanonical finite size scaling (MFSS) theory is developed, taking into account the following constraints (partly justified above):

(i) consistence with the canonical finite size scaling (CFSS) theory

(ii) power law behaviour of the finite system magnetization and susceptibility

(iii) finite system critical exponents which do not depend on the system size and which are not necessarily identical to those of the infinite system

\section{Microcanonical finite-size scaling (MFSS)}

In the vicinity of a critical point $\left(t, h, \varepsilon^{*}, m \approx 0\right)$, the thermodynamic potentials can be divided into a singular part (superscript ${ }^{*}$ ) which describes the non-analytic behaviour, and a regular part (subscript reg). For the free energy density and the specific entropy this reads:

$$
\begin{aligned}
& g(t, h)=g^{*}(t, h)+g_{\text {reg }}(t, h), \quad t:=\frac{T-T_{c}}{T_{c}}, \quad \begin{array}{l}
T_{c} \text { critical temperature } \\
h \text { magnetic field }
\end{array} \\
& s\left(\varepsilon^{*}, m\right)=s^{*}\left(\varepsilon^{*}, m\right)+s_{r e g}\left(\varepsilon^{*}, m\right) \quad, \quad \varepsilon^{*}:=\frac{\varepsilon-\varepsilon_{c}}{\left|\varepsilon_{c}\right|}, \quad \varepsilon_{c} \text { critical enthalpy }
\end{aligned}
$$

It can be shown (see e.g. [4] and references therein) that in the TDL the singular parts of the various potentials are homogeneous functions (cf. Eqs. (6), (7) in Fig. 2) and all critical exponents can be expressed in terms of the degrees of homogeneity $\left(a_{\varepsilon}=(1-\alpha) /(2-\alpha), a_{m}=1 /(\delta+1), a_{t}=1-a_{\varepsilon}\right.$, $\left.a_{h}=1-a_{m}\right)$. The equivalence of ensembles is valid only in the TDL. Hence, the thermodynamic potentials of finite systems have to be classified as canonical or microcanonical quantities (cf. [2], [6]). Starting point for the CFSS is the so-called "CFSS assumption" [7]: For large but finite systems, $g_{c}^{*}\left(t, h, L^{-1}\right)$ is a homogeneous function in accordance with Eq. (8).

We have shown elsewhere [6] that the MFSS assumption (9) entails (8), whereas proof of the reverse is more difficult. Note that Eq. (9) even accounts for the possibility of $s_{m}^{*}$ showing no system size dependence at all; nevertheless, this case results in an $L$-dependence of $g_{c}^{*}$ and in CFSS. In the rest of this paper, we will discuss the consequences of the MFSS assumption which states that:

$$
\begin{aligned}
& s_{m}^{*}\left(\varepsilon^{*}, m, L^{-1}\right) \text { is a homogeneous function } \\
& \text { of its arguments (cf. Fig. 2, Eq. (9)) }
\end{aligned}
$$

From the MFSS assumption (9) and Eqs. (11) and (2), the MFSS relations of the magnetization and the susceptibility are derived easily [3]:

$$
m_{h=0}^{*}\left(\varepsilon^{*}, L^{-1}\right)=\lambda^{-a_{m}} m_{h=0}^{*}\left(\lambda^{a_{\varepsilon}} \varepsilon^{*}, \lambda^{1 / d} L^{-1}\right) \stackrel{\lambda=L^{d}}{=} L^{-d a_{m}} \Phi_{m^{*}}\left(L^{d a_{\varepsilon}} \varepsilon^{*}\right)
$$


(6) $g^{*}(t, h)=\lambda^{-1} g^{*}\left(\lambda^{a} t t, \lambda^{a} h h\right)$

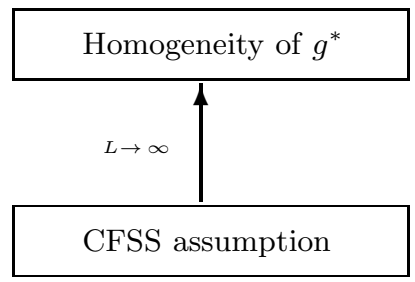

(8)

$$
\lambda^{-1} g_{c}^{*}\left(\lambda^{a} t, \lambda^{a} h h, \lambda^{1 / d} L^{-1}\right)
$$

Canonical Finite $\underline{\text { Size }} \underline{\text { Scaling }}$
(7) $s^{*}\left(\varepsilon^{*}, m\right)=\lambda^{-1} s^{*}\left(\lambda^{a} \varepsilon \varepsilon^{*}, \lambda^{a} m m\right)$
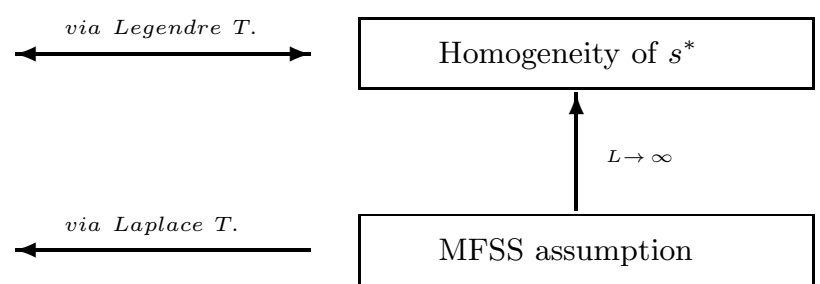

(9) $s_{m}^{*}\left(\varepsilon^{*}, m, L^{-1}\right)=$

$$
\lambda^{-1} s_{m}^{*}\left(\lambda^{a} \varepsilon \varepsilon^{*}, \lambda^{a} m m, \lambda^{1 / d} L^{-1}\right)
$$

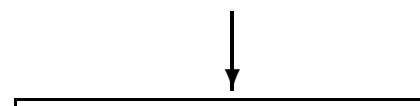

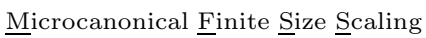

Fig. 2. Homogeneity relations (valid $\forall \lambda>0$ ) for the singular parts of the free energy and the entropy for finite (Eqs. (8),(9)) as well as infinite systems (Eqs. (6),(7)). In the TDL, a Legendre transform connects the homogeneity of $g^{*}$ to the homogeneity of $s^{*}$ (see Ref. [- [assumption is a consequence of the MFSS assumption, i.e., MFSS is consistent with CFSS 6].

$\chi_{T ; h=0}^{*}\left(\varepsilon^{*}, L^{-1}\right)=\lambda^{1-2 a_{m}} \chi_{T ; h=0}^{*}\left(\lambda^{a_{\varepsilon}} \varepsilon^{*}, \lambda^{1 / d} L^{-1}\right) \stackrel{\lambda=L^{d}}{=} L^{d\left(1-2 a_{m}\right)} \Phi_{\chi^{*}}\left(L^{d a \varepsilon} \varepsilon^{*}\right)$

where the $\Phi_{i}$ are so-called MFSS functions which describe the behaviour of the magnetization and the susceptibility of finite systems in the vicinity of the critical point $\varepsilon_{c}$ of the infinite system.

MFSS of the transition point: From Figs. 1a/c, it can be deduced that the MFSS function of the magnetization $\Phi_{m}(x)$ is zero for $L^{d a_{\varepsilon}} \varepsilon^{*}=: x \geq x_{T}$, where $x_{T}$ determines the magnetic transition point $\varepsilon_{T}(L)$ for finite systems:

$x_{T}=L^{d a_{\varepsilon}} \frac{\varepsilon_{T}(L)-\varepsilon_{c}}{\left|\varepsilon_{c}\right|} \Longleftrightarrow \varepsilon_{T}(L)=\varepsilon_{c}\left(1+\tilde{x}_{T} L^{-d a_{\varepsilon}}\right), \tilde{x}_{T}:=\frac{\varepsilon_{c}}{\left|\varepsilon_{c}\right|} x_{T}(12)$

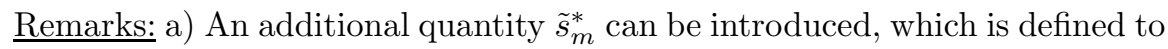
be the singular part of the entropy, written in terms of the reduced enthalpy $\tilde{\varepsilon}(L):=\left(\varepsilon-\varepsilon_{T}(L)\right) /\left|\varepsilon_{T}(L)\right|$. 
Using Eq. (12), the thus defined entropy $\tilde{s}_{m}^{*}$ can be shown to possess the same degrees of homogeneity as $s_{m}^{*}$ :

$$
\begin{aligned}
& \tilde{s}_{m}^{*}\left(\tilde{\varepsilon}, m, L^{-1}\right)=\lambda^{-1} \tilde{s}_{m}^{*}\left(\lambda^{a_{\varepsilon}} \tilde{\varepsilon}, \lambda^{a_{m}} m, \lambda^{1 / d} L^{-1}\right), \text { where } \\
& \tilde{s}_{m}^{*}\left(\tilde{\varepsilon}, m, L^{-1}\right):=s_{m}^{*}\left(\varepsilon^{*}=\tilde{\varepsilon}+x_{T} L^{-d a_{\varepsilon}}, m, L^{-1}\right) .
\end{aligned}
$$

Starting from expression (13), MFSS relations can be derived for the magnetization and the susceptibility. They illustrate the system size dependence of the behaviour of $m_{h=0}$ and $\chi_{T ; h=0}$ in the vicinity of the transition point $\varepsilon_{T}(L)$ of the finite system:

$$
\begin{aligned}
& \tilde{m}_{h=0}\left(\tilde{\varepsilon}, L^{-1}\right)=\lambda^{-a_{m}} \tilde{m}_{h=0}\left(\lambda^{a_{\varepsilon}} \tilde{\varepsilon}, \lambda^{1 / d} L^{-1}\right) \stackrel{\lambda=L^{d}}{=} L^{-d a_{m}} \Phi_{\tilde{m}}\left(L^{d a_{\varepsilon}} \tilde{\varepsilon}\right) \\
& \tilde{\chi}_{T ; h=0}\left(\tilde{\varepsilon}, L^{-1}\right)=\lambda^{1-2 a_{m}} \tilde{\chi}_{T ; h=0}\left(\lambda^{a_{\varepsilon}} \tilde{\varepsilon}, \lambda^{1 / d} L^{-1}\right) \stackrel{\lambda=L^{d}}{=} L^{d\left(1-2 a_{m}\right)} \Phi_{\tilde{\chi}}\left(L^{d a_{\varepsilon}} \tilde{\varepsilon}\right)
\end{aligned}
$$

b) Numerical data suggest power law behaviour for both the magnetization and the susceptibility of finite systems. Therefore, the MFSS functions $\Phi_{\tilde{m}}$ and $\Phi_{\tilde{\chi}}$ have to be power laws, governed by the respective finite system exponents which are not determined by the degrees of homogeneity of $s_{m}^{*}$ :

$$
\begin{aligned}
\Phi_{\tilde{m}}(x) & \propto(-x)^{\beta_{\varepsilon, L}} \quad \text { for } x \leq 0 \\
\Phi_{\tilde{\chi}}(x) & \propto|x|^{-\gamma_{\varepsilon, L}}
\end{aligned}
$$

c) $g_{c}^{*}$ is analytic for all finite $L$ and shows non-analyticities only in the TDL. To the best of our knowledge, no proof exists that $s_{m}^{*}$ is analytic for finite systems. Depending on the values of $\beta_{\varepsilon, L}$ and $\gamma_{\varepsilon, L}$, Eqs. (16) and (17) imply the possibility for $s_{m}^{*}$ to be either an analytic or a non-analytic function. This means that even for a completely analytic entropy, non-analyticities can occur for the microcanonical magnetization and susceptibility.

d) Note that it is indeed possible to explicitly construct a function $\tilde{s}_{m}^{*}$ which complies with the requirements (i)-(iii) stated at the end of the introduction. This explicit form is quite informative with respect to the "sudden" change of the exponents from their finite system values towards their values in the TDL, but has to be discussed elsewhere [6].

\section{Conclusion}

A MFSS theory has been developed in accordance with the demanded properties (i)-(iii) stated above. Amazingly, although the scaling laws (7) and (9) comprise identical degrees of homogeneity $a_{\varepsilon}$ and $a_{m}$ for the singular parts of the entropy of the finite and infinite system respectively, they nevertheless can account for power law behaviour with different exponents $\beta_{\varepsilon, L}, \gamma_{\varepsilon, L}$ for finite and $\beta_{\varepsilon, \infty}, \gamma_{\varepsilon, \infty}$ for infinite systems. As canonical potentials emerge from the microcanonical ones basically by means of a Laplace transform, it is to be expected that the smoothing properties of this integral transform cause MFSS to be applicable for smaller systems than CFSS. 


\section{References}

1. In the case of an Ising system, the specific enthalpy $\varepsilon$ is given by the interaction energy: $\varepsilon:=-L^{-d} J \sum_{\langle i, j\rangle} S_{i} S_{j}$, i.e., $\varepsilon$ does not contain the field dependent term $h \sum_{i} S_{i}$ of the Ising Hamiltonian.

2. M. Kastner, M. Promberger, A. Hüller, in "Computer Simulation Studies in Condensed Matter Physics XI", Eds. D.P. Landau, H.-B. Schüttler, Heidelberg (1998)

3. The zero-field magnetization and the susceptibility can be expressed in terms of $\varepsilon, \varepsilon^{*}$ or $\tilde{\varepsilon}$. The corresponding functions are denoted by $m, \chi ; m^{*}, \chi^{*}$ or $\tilde{m}, \tilde{\chi}$.

4. C. DiCastro, G. Jona-Lasinio, in "Phase Transitions and Critical Phenomena" Vol. 6, Eds. C. Domb, M.S. Green, London (1976)

5. H.E. Stanley, "Introduction to Phase Transitions and Critical Phenomena", Appendix C, Oxford (1971)

6. M. Kastner, M. Promberger, A. Hüller, to be published

7. M.E. Fischer, M.N. Barber, Phys. Rev. Lett. 28, 1516 (1972) 